\title{
A Postal Screener for Pain and Need for Treatment in Older Persons in Primary Care
}

\author{
Margot W. M. de Waal, PhD, Wendy P. J. den Elzen, PhD, Wilco P. Achterberg, MD, PhD, \\ Jacobijn Gussekloo, MD, PhD, and Jeanet W. Blom, MD, PhD
}

OBJECTIVES: To test the Pain intensity, Enjoyment in life, General activity questionnaire (PEG) as a postal screener for pain in older persons.

DESIGN: Population-based survey. Postal screening questionnaires followed by an interview of a sample of participants.

SETTING: Family practices.

PARTICIPANTS: Persons aged 75 and older $(\mathrm{N}=243$; 95 interviewed).

MEASUREMENTS: Screening included the PEG, a threeitem abbreviated version of the Brief Pain Inventory (BPI), plus an additional question on treatment need. Pain severity and related interference was assessed (BPI) during the interview, as was the current (need for) pain treatment.

RESULTS: The median PEG score of the 243 persons participating in the screening (response $76 \%$ ) was 2.0 (interquartile range $0-4.7)$. Seventy-nine $(35 \%)$ had moderate to severe pain (PEG score $\geq 4$ ), of whom $56 \%$ reported current pain treatment and $15 \%$ stated that they might ask for help. For a PEG score cutoff of 4 or greater, sensitivity was 0.81 and specificity was 0.78 to find scores of 4 or greater on one or both BPI subscales during the interview. For the question on need for treatment, replies on the screener and the interview were not always consistent. Of the 43 interviewed participants with a PEG score of 4 or greater, $60 \%$ received treatment. Of the 17 without current pain treatment, 10 still reported pain, three of whom said that they might ask for help.

CONCLUSION: The PEG can be used as a postal screener to detect the presence of pain in older persons, but treatment needs cannot be established using the PEG alone or in combination with a simple additional question. J Am Geriatr Soc 2014.

From the Department of Public Health and Primary Care, Leiden University Medical Center, Leiden, the Netherlands.

Address correspondence to Margot W. M. de Waal, Department of Public Health and Primary Care, Leiden University Medical Center, PO Box 9600, 2300 RC Leiden, the Netherlands.

E-mail: m.w.m.de_waal@lumc.nl

DOI: $10.1111 /$ jgs.13064
Key words: pain; screening; old age

$\mathbf{F}$ amily physicians are faced with a growing population of older adults. For example, in the European Union, the percentage of people aged 65 and older is expected to rise from $18 \%$ in 2013 to $29 \%$ in $2080 .{ }^{1}$ One of the challenges associated with older adults is management of pain. $^{2-4}$ Pain negatively affects all domains of health, including mobility and physical functioning, cognitive functioning, mood, and sleep-wake rhythm..$^{5-10}$ Because pain makes maintaining an independent daily life and sustaining social contacts difficult, it often leads to poor quality of life.

Family physicians perceive chronic nonmalignant pain to be a challenge and consider chronic pain and its effect on quality of life to be underassessed in primary care. ${ }^{4,11}$ Older adults with pain may be less likely to report their pain symptoms to physicians than the younger, workingage population. One explanation for this is that the current generation of older people appear to be stoic about reporting pain sensations. ${ }^{12,13}$ Another explanation is decreasing cognitive functioning and, therefore, decreasing ability to communicate pain. ${ }^{3,14}$ Studies that quantify under-reporting are scarce. Forty-six percent of people aged 50 and older and $50 \%$ of those aged 75 and older with chronic knee pain had consulted their family physician regarding this symptom. ${ }^{15}$ A Swedish study showed an increase in pain but a decrease in pain medication with age, raising concerns about the spontaneous reporting of pain of older adults and the ability of physicians to detect this almost "hidden" problem. ${ }^{16}$

Without explicit questioning, older people are likely to underreport chronic pain, so a more-proactive approach is recommended. ${ }^{5,17,18}$ To tackle this problem, a suitable and feasible method of screening for pain should be developed and validated. Multidimensional pain measures, such as the Brief Pain Inventory (BPI), are widely used in specialized pain centers and research settings, but the BPI is too comprehensive for screening purposes in primary care, 
where a brief instrument would be more feasible. A threeitem, abbreviated version of the BPI, assessing pain intensity and functional interference (Pain intensity, Enjoyment in life, General activity; PEG) has been developed for use in primary care. ${ }^{19}$ There is strong evidence of its reliability, construct validity, and responsiveness when used in interviews with individuals in primary care with chronic musculoskeletal pain (mean age 59) and veterans recruited from ambulatory care (mean age 63).

The present study aimed to test the PEG using a postal questionnaire for pain screening in people aged 75 and older in primary care in terms of the presence of pain, whether or not it interferes with daily life and experienced treatment needs. Because the acceptance of an unsolicited treatment offer after screening is always a concern, an additional question was added asking about the need for treatment, because the PEG does not include such an item.

\section{METHODS}

\section{Participants}

This study was conducted within the larger Integrated Systematic Care for Older People (ISCOPE) study, in which data were obtained on demographic and clinical characteristics of individuals in primary care aged 75 and older living in the community and in care homes. The overall aim of the ISCOPE study was to assess the efficacy of a simple structural monitoring system to detect deterioration in the functional, somatic, mental, or social health of individuals aged 75 and older, followed by the execution of a care plan for those with a combination of somatic, functional, mental, and social problems. ${ }^{21}$

The ISCOPE study took place in 59 family practices in an urban area in the western part of the Netherlands. At baseline, screening questionnaires were sent to all persons aged 75 and older. The physicians excluded individuals with a life expectancy of $<3$ months. Participants were asked to fill in the questionnaire at baseline and again at 6 and 12 months. Of the older persons returning the screening questionnaire, a random sample was invited for an interview at home at baseline and 12 months after baseline. The interviews covered additional information on sociodemographic characteristics, chronic diseases, use of healthcare, functioning, and quality of life.

This article presents the data of an additional observational study on pain screening that was performed in three of the 59 family practices at the 12-month follow-up assessment of the ISCOPE study. For this, questions on pain and treatment were added to the postal screening (including the PEG) and the interviews.

All participants provided written informed consent. The medical ethical committee of the Leiden University Medical Center approved the ISCOPE study (NTR1946).

\section{Measures}

\section{Postal Screening}

For the present cross-sectional study, the screening included two questionnaires: the PEG with the additional question about treatment needs and the ISCOPE screening questionnaire.

The PEG is a three-item scale assessing pain intensity $(\mathrm{P})$, interference with enjoyment of life $(\mathrm{E})$, and interference with general activity $(\mathrm{G})$ during the past week. ${ }^{19}$ Each item is scored on a numeric scale from 0 to 10 ; a total score is computed by taking the mean score on all items (range 0-10). A question was added about the individual's treatment needs, in which four options concerning asking for advice or help for pain from a family physician or other caregiver were given. This question was constructed based on Prochaska's Stages of Change model, which describes the steps in the process of behavior change. ${ }^{20} \mathrm{It}$ indicates whether individuals might be motivated for treatment (ready to change).

The ISCOPE screening questionnaire contains items on somatic, functional, mental, and social health (4-7 questions per domain). If participants scored positive on two or more items, this domain was classified as a problem domain. Pain is excluded from scoring in all domains.

\section{Interview}

All participants with problems ( $\geq 2$ positive answers) on three or four domains on the ISCOPE questionnaire were invited for an interview; this group was supplemented with a random sample of $60 \%$ of participants with problems on two domains and a random sample of $15 \%$ of participants with problems on none or one of the domains. ${ }^{21}$

The selected participants were interviewed with structured questionnaires about the presence and intensity of pain and its interference with daily life, use of pain treatment and pain medication, living arrangements, cognition, presence of chronic diseases, and self-reported use of health care.

The BPI short form includes four items on pain intensity (average, current, least, worst) and seven items on pain interference (interference with general activity, mood, walking, work including housework, relationships with others, sleep, enjoyment of life). ${ }^{22,23}$ For each scale, a total score is computed by taking the mean score of all items (range 0-10). For this study, the time frame was adapted from "today" to the "past week." The official lead-in question of the BPI was extended with an additional sentence that asked about the use of pain medication or a change in lifestyle to relieve pain. Four extra questions about concerns about taking medication (taken from the long version of the BPI) were also added, as well as questions about knowledge of pain and treatment by the family physician or other caregivers. ${ }^{24}$

Cognition was assessed using the Mini-Mental State Examination. $^{25}$

\section{Analyses}

Descriptive statistics on screening outcomes of the total sample were determined. Descriptive statistics were also determined in the sample of interviewed participants, and the PEG was validated by analyzing Spearman correlations between total PEG score and BPI subscale scores. 
Because there is no known cutoff score (and no criterion standard) for the PEG, participants were categorized into three groups according to score $(0.0,0.1-3.9, \geq 4.0)$. Median scores and interquartile range (IQR) on both BPI subscales were calculated for these PEG groups, and differences between groups were tested using a nonparametric test. Percentages were calculated for dichotomous measures, and differences between groups were tested using the chi-square test.

\section{RESULTS}

In the three family practices, response at baseline in the ISCOPE study was $66 \%$ (354 of 528), and loss to followup after 12 months was $9 \%$ (33 of 354). Of the final sample of 321 participants who received the screening questionnaires at 12 months, $76 \% \quad(\mathrm{n}=243)$ fully completed the pain screening questionnaire.

\section{Pain Screening and Participants' Experienced Need for Treatment}

Table 1 shows the characteristics of the participants and their screening outcomes. Mean age of participants was $82.4 \pm 4.3 ; 55 \%$ were female. General screening showed that $28 \%$ of participants had problems in three or more domains. PEG scores were 0.0 in $32 \%, 0.1$ to 3.9 in $35 \%$, and 4.0 or higher in $33 \%$. The median PEG score was 2.0 (IQR $0 ; 4.7)$.

Overall, $30.8 \%$ reported current treatment or use of medication for pain, and $9.7 \%$ reported that they might

\begin{tabular}{lc}
\hline \multicolumn{1}{c}{ Table 1. } & Participant Characteristics $(\mathbf{N}=243)$ \\
\hline \multicolumn{1}{c}{ Characteristic } & \multicolumn{1}{c}{ Value } \\
\hline Female, $n(\%)$ & $134(55.1)$ \\
Age, $n(\%)$ & \\
$75-79$ & $85(35.0)$ \\
$80-84$ & $101(41.6)$ \\
$\geq 85$ & $57(23.5)$ \\
General screening on functioning, $n$ (\%) & \\
Problem domains & \\
Type & \\
Somatic & $127(53.4)$ \\
Functional & $62(26.1)$ \\
Mental & $114(47.9)$ \\
Social & $78(32.8)$ \\
$\geq 3$ domains & $67(27.6)$ \\
Pain screening & \\
PEG items, median (IQR) & \\
Average pain intensity & $3.0(0-5)$ \\
Interference with enjoyment in life & $1.0(0-5)$ \\
Interference with general activity & $1.0(0-5)$ \\
PEG score, median (IQR) & $2.0(0-4.7)$ \\
Participant's experienced need for treatment regarding pain, $\mathrm{n}(\%)$ \\
No need for help & $123(51.9)$ \\
Will not ask for help & $18(7.6)$ \\
Might ask for help & $23(9.7)$ \\
Current treatment or medication & $73(30.8)$ \\
\hline
\end{tabular}

$\mathrm{IQR}=$ interquartile range.

PEG = Pain intensity, Enjoyment in life, General activity questionnaire (range 0-10).

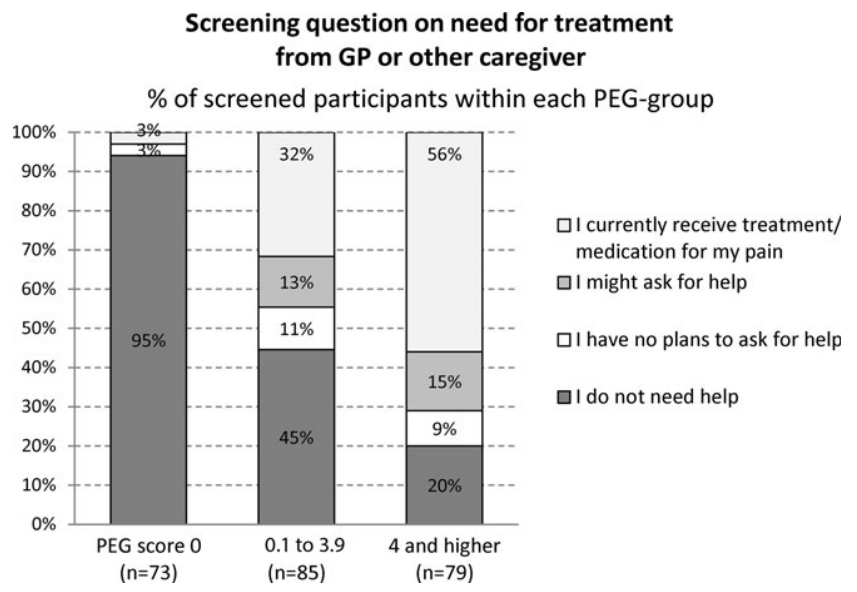

Figure 1. Participants' experienced need for treatment regarding pain according to Pain intensity, Enjoyment in life, General activity (PEG) score (range $0-10)(\mathrm{N}=237$, missing for $\mathrm{n}=6) . \mathrm{GP}=$ general practitioner.

ask for help. Figure 1 presents the current treatment and treatment needs for the three PEG groups. Participants with a PEG score of 4.0 and greater were more likely to receive pain treatment $(56 \%)$ than participants with a PEG score of 0.1 to $3.9(32 \%$, chi-square test $P=.002)$, although the percentage of participants who might ask for help in the future was similar in those with a PEG score of 4.0 and greater $(15 \%)$ and 0.1 to $3.9(13 \%$, chi-square test $P=.68$ ).

\section{Interview Results: Presence of Pain, Treatment, and} Need for Treatment

A sample of 95 participants was interviewed. These participants indicated more problems on the ISCOPE screening questionnaire than the total sample $(40 \%$ had problems in $\geq 3$ domains). For six participants $(6 \%)$, cognition was low, with Mini-Mental State Examination scores $<22$. The main self-reported chronic diseases were osteoarthritis $(59 \%)$, heart failure $(44 \%)$, asthma or chronic obstructive pulmonary disease $(26 \%)$, osteoporosis $(23 \%)$, malignancy $(13 \%)$, and diabetes mellitus (15\%). According to the BPI, 50 participants $(53 \%)$ reported having had pain in the previous week. The most frequently mentioned pain locations were the lower extremities (72\%), back (46\%), and upper extremities $(40 \%) ; 44 \%$ of participants reported more than one location.

\section{Validation of the PEG with the BPI}

PEG scores correlated highly with the BPI subscales severity (correlation coefficient $(r)=0.668, P<.001$ ) and interference $(r=0.789, P<.001)$. Table 2 shows the presence of pain and subscale scores of the BPI for groups according to PEG score. Participants with PEG scores of 4.0 and higher during screening reported pain in $81 \%$ of the cases, with a median BPI severity score of 4.5 (IQR 2.2-6.0) and a median BPI interference score of 3.9 (IQR 1.0-5.9). The sensitivity of a PEG score cutoff of 4 or greater to detect BPI severity or interference scores of 4 or greater was 0.81 , the specificity was 0.78 , and the positive 
Table 2. Reported Pain and Treatment of Interviewed Participants $(\mathrm{n}=95)$ According to Total Pain intensity, Enjoyment in life, General activity (PEG) Score

\begin{tabular}{|c|c|c|c|}
\hline \multirow[b]{2}{*}{ Characteristic } & \multicolumn{3}{|c|}{ Screening Total PEG Score } \\
\hline & $0.0, n=26$ & $0.1-3.9, n=26$ & $\geq 4.0, n=43$ \\
\hline Pain present last week, n (\%) & $0(0)$ & $15(58)$ & $35(81)$ \\
\hline BPI severity subscale, median (IQR) & $0(0-0)$ & $2.3(0-4.8)$ & $4.5(2.2-6.0)$ \\
\hline BPI interference subscale, median (IQR) & $0(0-0)$ & $0(0-2.3)$ & $3.9(1.0-5.9)$ \\
\hline No treatment, $\mathrm{n}(\%)$ & $25(96)$ & $17(65)$ & $17(40)$ \\
\hline Pain, $\mathrm{n}$ & 0 & 6 & 10 \\
\hline No pain, $\mathrm{n}$ & 25 & 11 & 7 \\
\hline \multicolumn{4}{|l|}{ Medication for pain, $\mathrm{n}(\%)^{\mathrm{d}}$} \\
\hline Prescribed medication & $1(4)$ & $6(23)$ & $23(53)$ \\
\hline Only over-the-counter medication & $0(0)$ & $4(15)$ & $8(19)$ \\
\hline
\end{tabular}

BPI = Brief Pain Inventory (range 0-10).

${ }^{a}$ Healthcare professional: specialist, family physician, or physiotherapist.

${ }^{\mathrm{b}}$ Twenty-five of 26 participants with a PEG score $\geq 4.0$ reported pain.

cPearson chi-square test $P=.04$, degree of freedom $(d f)=1$ when comparing groups with a score of $0.1-3.9$ and $\geq 4.0$.

${ }^{\mathrm{d}}$ Pearson chi-square test $P=.03, d f=2$ when comparing groups with a score of $0.1-3.9$ and $\geq 4.0$.

predictive value was 0.70 . The sensitivity of a PEG score of 4 or greater to detect BPI scores of 5 or greater was 0.82 , the specificity was 0.70 , and the positive predictive value was 0.53 .

\section{Reported (Need for) Treatment}

Table 2 presents the treatment as the interviewed participants reported it. Treatment from healthcare professionals included medication and other therapies (e.g., physiotherapy).

Of the participants with PEG scores of 4.0 or higher during screening, $60 \%(\mathrm{n}=26)$ received treatment from healthcare professionals. Of the 17 participants without treatment, 10 reported pain, with a mean BPI interference score of 3 (range 1-5). When PEG scores were between 0.1 and $3.9,35 \%(\mathrm{n}=9)$ of the participants received treatment from healthcare professionals, and six of the 17 participants without treatment reported pain; these six participants had a mean BPI interference score of 2 (range 1-3).

These 10 and six patients without treatment but with pain were asked whether they had plans to ask for help from their family physician; three indicated that they might ask for help. Thirteen stated that their family physician had been informed about their pain, and 10 thought that nothing (more) could be done. Participants with a PEG score of 4.0 or greater and a PEG score of 0.1 to 3.9 with current pain (irrespective of present medication use) were asked whether they might need more or stronger medication; six of these 50 participants $(12 \%)$ replied "yes."

Of the interviewed participants with a PEG score of 4.0 or greater, $53 \%$ received prescribed medication; another $19 \%$ used over-the-counter medication. Participants with PEG scores between 0.1 and 3.9 used less medication (Table 2).

\section{Validation of Screening Question on Need for Treatment}

The replies to the question on the need for treatment from the screener were also compared with that from the interview, regardless of PEG score. Of the 15 interviewed participants who reported in the screener that they might ask for help, five reported no pain during the interview, and four reported receiving treatment from a healthcare professional for pain. The remaining six participants were asked whether they had plans to ask for help from their family physician; two said that they might ask for help, and four did not intend to ask for help.

\section{DISCUSSION}

Results of the present study with a postal screener for pain in a family practice population aged 75 and older confirmed previous studies that found that pain in older persons in primary care is highly prevalent. Thirty-three percent of participants reported moderate to severe pain during the last week (PEG total score $\geq 4.0$ ), and $35 \%$ reported mild pain (PEG total score 0.1-3.9). The threeitem PEG screening questionnaire appeared to be a valid instrument to identify those who are in pain. PEG scores were as good a reflection of pain severity and associated functioning as the BPI (construct validity).

Because the acceptance of an unsolicited treatment offer after screening is always a point of concern, an additional question on treatment needs was added to the postal screener. This revealed that most individuals with pain already received some form of treatment or medication, and only a minority reported a need for care. Of participants with a PEG score of 4.0 or greater, $56 \%$ reported current pain treatment or medication, and $15 \%$ indicated that they might ask for help in the future. When trying to validate the question, the need for treatment could not be 
confirmed because the replies on the screener and the interview were not always consistent.

In the group with a PEG score $<4.0$, a similar percentage reported that they might ask for help; the reason for this similar percentage is not clear.

Few studies have used postal questionnaires for specific types of pain (e.g., knee pain ${ }^{15}$ ) or a more-general health screening questionnaire that includes a single item on pain. Apparently, pain is not high on the list of screening conditions. It seems that general guidelines and experts do not consider pain to be the most relevant topic for screening for healthy or vulnerable older persons. ${ }^{26}$ One reason for this is that there is little evidence that screening will lead to better therapy and thus better health outcomes, although one study showed that treating individuals with pain detected using screening might be worthwhile. One study reported a modest but statistically significant improvement in a variety of outcome measures from a collaborative care intervention for chronic pain in primary care. ${ }^{27}$ Routine pain screening during consultations (pain as the fifth vital sign) was promoted in clinics for veterans in the United States using a numeric rating scale, but a medical record review showed that simply implementing the numeric rating scale for pain did not necessarily improve subsequent pain evaluation and management. $^{28}$

Do the current results suggest that postal screening for pain is useful? It was possible to detect who was in pain, but the need for treatment could not be fully determined. A postal screener alone is not enough to improve assessment and treatment. Most participants in pain had already had contact with a family physician or other caregiver. Therefore, more benefit might be expected from alertness, assessment, and clinical evaluation of pain during consultations. In general, it was found that communication of pain information by family physicians has some deficiencies. Assessment of pain information should be multidimensional and include eliciting pain treatment information as well as location and sensory aspects of pain information. ${ }^{12}$ For individuals with cognitive problems (e.g., dementia), assessment is more complicated and cannot rely on self-report alone. ${ }^{14}$ Education on assessment and treatment is important, and guidelines can provide this knowledge. ${ }^{18,29-31}$ European primary care physicians emphasized in a survey the necessity of training in the use of assessment tools, the appropriate prescription of strong opioids, and the development of guidelines. ${ }^{4}$

In this study, $56 \%$ of the participants with moderate or severe pain (PEG score $\geq 4.0$ ) reported current treatment, but $44 \%$ did not. From this latter group, one in three stated that they might ask for help. It is possible that participants had discussed their pain with a physician in the past but that the physician concluded that (at that time) nothing needed to be done (wait and see) or that nothing more could be done (no cure expected). Even though the pain was still present, the individuals might have given up or for some reason been waiting to visit the physician again. An often-stated reason for not asking or receiving help is ageism; individuals and physicians might see pain as a part of normal aging. 32
Alternatively, in a qualitative study on joint pain from osteoarthritis, the fact that arthritis was seen as a normal part of old age did not hamper professional consultation for new joint symptoms if necessary. ${ }^{33}$ It seems that most older adults are willing to seek help, but some may not find what they are looking for.

Qualitative research revealed that older adults are willing to try a variety of strategies to help manage persistent pain, although, on average, they view these strategies as only moderately helpful. ${ }^{34}$ Older adults rely more on strategies that can be self-administered and are reluctant to take medication. ${ }^{35}$ In line with this, a larger role for family physicians is suggested in discussing self-management with patients and providing guidance on the safety of specific self-care activities. ${ }^{36}$

It was possible to test the PEG in a family practice population aged 75 and older using only a few exclusion criteria. Because no criterion standard exists for pain, the well-known BPI and two cutoff points of the BPI 4 and 5 was used as the criterion standard. Higher cutoff scores would detect more-severe or more-interfering pain.

In the present study, there was no information from family physicians on the (need for) treatment of the participants. Thus, whether a new or different treatment or medication regime would be more effective, or even realistic, could not be evaluated.

Timely and adequate treatment for pain is important, because pain has many negative effects. This study shows that pain is highly prevalent and probably undertreated. The results indicate that screening for pain in primary care using a short postal questionnaire is feasible, although one written question is not enough to explore treatment needs; more benefit might be expected when the family physician assesses treatment needs explicitly during patient visits. Exploration of these treatment needs and the role of the family physician should be the subject of more in-depth study.

\section{ACKNOWLEDGMENTS}

The authors thank all the participants, family physicians, and research nurses (Jeanet van der Born, Astrid Cock, Anja Garstman, Myriam de Groot, Els de Haas, Ellen Koorte-kaas, Tineke Lacourt, Inge Mooyekind, Ingrid van Oor-schot, Wilma van Rijn, Mieke Slegt, Corrie Vlieland, Olga Weeda) who contributed to this research project.

Conflict of Interest: The editor in chief has reviewed the conflict of interest checklist provided by the authors and has determined that the authors have no financial or any other kind of personal conflicts with this paper.

This study was partly funded by ZonMw (Organization for Health Research and Development; ZonMw number 311060201).

Author Contributions: All authors discussed the results and commented on the manuscript. MWMdW, JWB: conception and design, acquisition of data, analysis and interpretation of data, drafting and revising the article. WPJdE, JG: conception and design, acquisition of data, revising the article. WPA: writing and revising the article.

Sponsor's Role: The sponsor had no role in the design, methods, subject recruitment, data collection, analysis, or preparation of the paper. 


\section{REFERENCES}

1. Eurostat 2013. Population Structure and Ageing [on-line]. Available at http://epp.eurostat.ec.europa.eu/statistics_explained/index.php/Population_structure_and_ageing Accessed August 11, 2014.

2. Chodosh J, Solomon DH, Roth CP et al. The quality of medical care provided to vulnerable older patients with chronic pain. J Am Geriatr Soc 2004;52:756-761.

3. Achterberg WP, Pieper MJ, van Dalen-Kok AH et al. Pain management in patients with dementia. Clin Interv Aging 2013;8:1471-1482.

4. Johnson M, Collett B, Castro-Lopes JM. The challenges of pain management in primary care: A pan-European survey. J Pain Res 2013;6: $393-401$.

5. American Geriatrics Society. Panel on Persistent Pain in Older Persons. The management of persistent pain in older persons. J Am Geriatr Soc 2002;50: S205-S224.

6. Dekker J, Boot B, van der Woude LH et al. Pain and disability in osteoarthritis: A review of biobehavioral mechanisms. J Behav Med 1992;15:189214.

7. Kendig H, Browning CJ, Young AE. Impacts of illness and disability on the well-being of older people. Disabil Rehabil 2000;22:15-22.

8. Landi F, Russo A, Liperoti $\mathrm{R}$ et al. Daily pain and functional decline among old-old adults living in the community: Results from the ilSIRENTE Study. J Pain Symptom Manage 2009;38:350-357.

9. Gambassi G. Pain and depression: The egg and the chicken story revisited. Arch Gerontol Geriatr 2009;49(Suppl 1):103-112.

10. Jakobsson U, Klevsgard R, Westergren A et al. Old people in pain: A comparative study. J Pain Symptom Manage 2003;26:625-636.

11. Gibson SJ. IASP global year against pain in older persons: Highlighting the current status and future perspectives in geriatric pain. Expert Rev Neurother 2007;7:627-635.

12. Abdulla A, Adams N, Bone M et al. Guidance on the management of pain in older people. Age Ageing 2013;42(Suppl 1):i1-i57.

13. Helme RD, Gibson SJ. The epidemiology of pain in elderly people. Clin Geriatr Med 2001;17:417-431.

14. Herr K. Pain assessment strategies in older patients. J Pain 2011;12:S3S13.

15. Jinks C, Jordan K, Ong BN et al. A brief screening tool for knee pain in primary care (KNEST). 2. Results from a survey in the general population aged 50 and over. Rheumatology (Oxford) 2004;43:55-61.

16. Jakobsson U. The epidemiology of chronic pain in a general population: Results of a survey in southern Sweden. Scand J Rheumatol 2010;39:421429.

17. Royal College of Physicians, British Geriatrics Society and British Pain Society.The Assessment of Pain in Older People: National Guidelines. Concise Guidance to Good Practice Series, No 8. London: RCP, 2007.

18. Achterberg WP, de Ruiter CM, de Weerd-Spaetgens CM et al. Multidisciplinary guideline 'Recognition and treatment of chronic pain in vulnerable elderly people'. Ned Tijdschr Geneeskd 2012;155:A4606.
19. Krebs EE, Lorenz KA, Bair MJ et al. Development and initial validation of the PEG, a three-item scale assessing pain intensity and interference. J Gen Intern Med 2009;24:733-738.

20. Dijkstra A. The validity of the stages of change model in the adoption of the self-management approach in chronic pain. Clin J Pain 2005;21:27-37.

21. Poot AJ, den Elzen WP, Blom JW et al. Level of satisfaction of older persons with their general practitioner and practice: Role of complexity of health problems. PLoS One 2014;9:e94326.

22. Cleeland CS, Ryan KM. Pain assessment: Global use of the Brief Pain Inventory. Ann Acad Med Singapore 1994;23:129-138.

23. Tan G, Jensen MP, Thornby JI et al. Validation of the Brief Pain Inventory for chronic nonmalignant pain. J Pain 2004;5:133-137.

24. MD Anderson Cancer Center. Brief Pain Inventory [on-line]. Available at http://www.mdanderson.org/education-and-research/departments-programsand-labs/departments-and-divisions/symptom-research/symptom-assessmenttools/brief-pain-inventory.html Accessed March 3, 2011.

25. Folstein MF, Folstein SE, McHugh PR. 'Mini-mental state'. A practical method for grading the cognitive state of patients for the clinician. J Psychiatr Res 1975;12:189-198.

26. Drewes YM, Gussekloo J, van der Meer V et al. Assessment of appropriateness of screening community-dwelling older people to prevent functional decline. J Am Geriatr Soc 2012;60:42-50.

27. Dobscha SK, Corson K, Perrin NA et al. Collaborative care for chronic pain in primary care: A cluster randomized trial. JAMA 2009;301:12421252.

28. Zubkoff L, Lorenz KA, Lanto $\mathrm{AB}$ et al. Does screening for pain correspond to high quality care for veterans? J Gen Intern Med 2010;25:900-905.

29. Kress HG. The importance of putting pain on the curricula in medical schools in Europe. J Pain Palliat Care Pharmacother 2013;27:182-184.

30. Niv D, Devor M. Position paper of the European Federation of IASP Chapters (EFIC) on the subject of pain management. Eur J Pain 2007;11:487489.

31. Institute of Medicine. Relieving Pain in America: A Blueprint for Transforming Prevention, Care, Education, and Research. Washington, DC: The National Academies Press, 2011.

32. Brown D. A literature review exploring how healthcare professionals contribute to the assessment and control of postoperative pain in older people. J Clin Nurs 2004;13:74-90.

33. Grime J, Richardson JC, Ong BN. Perceptions of joint pain and feeling well in older people who reported being healthy: A qualitative study. $\mathrm{Br} \mathrm{J}$ Gen Pract 2010;60:597-603.

34. Kemp CA, Ersek M, Turner JA. A descriptive study of older adults with persistent pain: Use and perceived effectiveness of pain management strategies [ISRCTN11899548]. BMC Geriatr 2005;5:12.

35. Lansbury G. Chronic pain management: A qualitative study of elderly people's preferred coping strategies and barriers to management. Disabil Rehabil 2000;22:2-14.

36. MacKichan F, Paterson C, Britten N. GP support for self-care: The views of people experiencing long-term back pain. Fam Pract 2013;30:212-218. 